\title{
Large Coded Aperture Mask for Spaceflight Hard X-ray Images
}

\author{
Danielle N. Vigneau*, David W. Robinson** \\ NASA Goddard Space Flight Center, Greenbelt, MD
}

\author{
Keywords: Coded Aperture Mask, Swift, Burst Alert Telescope, Hard X-rays
}

\begin{abstract}
The 2.6 square meter coded aperture mask is a vital part of the Burst Alert Telescope on the Swift mission. A random, but known pattern of more than 50,000 lead tiles, each $5 \mathrm{~mm}$ square, was bonded to a large honeycomb panel which projects a shadow on the detector array during a gamma ray burst. A two-year development process was necessary to explore ideas, apply techniques, and finalize procedures to meet the strict requirements for the coded aperture mask. Challenges included finding a honeycomb substrate with minimal gamma ray attenuation, selecting an adhesive with adequate bond strength to hold the tiles in place but soft enough to allow the tiles to expand and contract without distorting the panel under large temperature gradients, and eliminating excess adhesive from all untiled areas. The largest challenge was to find an efficient way to bond the $>50,000$ lead tiles to the panel with positional tolerances measured in microns. In order to generate the desired bondline, adhesive was applied and allowed to cure to each tile. The "pre-cured" tiles were located in a tool to maintain positional accuracy, wet adhesive was applied to the panel, and it was lowered to the tile surface with synchronized actuators. Using this procedure, the entire tile pattern was transferred to the large honeycomb panel in a single bond. The pressure for the bond was achieved by enclosing the entire system in a vacuum bag. Thermal vacuum and acoustic tests validated this approach. This paper discusses the methods, materials, and techniques used to fabricate this very large and unique coded aperture mask for the Swift mission.
\end{abstract}

\section{Introduction and Background}

The Swift satellite is designed to detect and observe Gamma-Ray Bursts. ${ }^{1}$ One of the three instruments on Swift is the Burst Alert Telescope, or BAT. Its function is to detect Gamma Ray Bursts in a wide field of view and to report their locations to the spacecraft so that it may immediately point the other two onboard narrow-field telescopes to the burst for closer observation at UV and X-ray wavelengths. BAT's large $\left(5243 \mathrm{~cm}^{2}\right)$ detector array detects $\mathrm{X}$-rays (15-150 keV range) that pass through a very large $\left(2.6 \mathrm{~m}^{2}\right)$ coded aperture mask and project a shadow image on the detector array.

BAT Mask and Mask Support Structure

$\mathrm{X}$-rays photons with energy greater than about $10 \mathrm{keV}$ cannot be focused with grazing incidence mirrors. BAT is required to observe X-rays at energies between $15-150 \mathrm{keV}$ over a large field of view. To do this, BAT uses a coded aperture mask in combination with a detector array. Coded aperture mask technology has been in the literature since the 1960s and many have flown on space telescopes. An ideal mask consists of a random but known pattern of transparent and opaque zones. When X-rays, such as from a Gamma Ray Burst, penetrate the mask, a shadow pattern of these zones is cast upon the detector array below. By analyzing the patterns seen by the detectors, a vector can be drawn pointing to the origin of the X-rays. Many papers have been written about this technique; sources are provided in the reference section for the interested reader. ${ }^{2}$

BAT's coded aperture mask consists of about 50,000 lead tiles, $5 \times 5 \times 1$ m, arranged on a $2.4 \times 1.9$ meter honeycomb panel in a random, but known, pattern. It is the largest such device ever built. Early in the BAT project, the team realized that considerable research and development would be required to build the mask due to its large size. It was difficult to find other mask designs suitable for scaling up to the size required for Swift. One example is INTEGRAL's JEM-X mask. ${ }^{3}$ The $0.2 \mathrm{~m}^{2}$ JEM-X

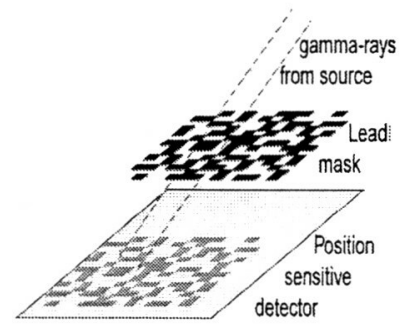


mask was designed for a similar energy range (5-60 keV) as BAT and used a single $0.5 \mathrm{~mm}$ thick tungsten sheet with 5,800 hexagonal holes cut from it in a uniformly redundant array pattern. The sheet was placed on a spider-web shaped titanium stiffener ring, and the entire assembly weighed about $6.85 \mathrm{~kg}$. Scaling this design up thirteen times to the size of BAT's mask would have exceeded the mass requirements as well as created difficult thermal expansion and stiffness issues. Clearly a different concept was required for BAT.

After two years of intense effort, techniques were developed to overcome the challenges and meet BAT's stringent requirements. This paper will focus on those engineering challenges which include precisely positioning $\sim 50,000$ lead tiles on the honeycomb panel and ensuring they withstand the harsh environments of rocket launch low earth orbit.

\section{BAT Coded Aperture Mask Requirements}

There were many requirements levied on BAT's mask from the science and engineering disciplines. The size of the . mask was to be as large as possible and still fit into the Delta II rocket fairing. In an attempt to detect an average of one gamma ray burst per day, the $50 \%$ coded field of view was set at 2.1 steradians and the fully coded field of view at 1.4 sr. To cast a distinct shadow on the detector array, the mask panel needed to allow $35 \%$ transmission of X-rays at 10 $\mathrm{keV}$ while the tiles blocked about $90 \%$ of the X-rays at $150 \mathrm{keV}$. Each of the $5 \times 5 \times 1 \mathrm{~mm}$ lead tiles had to be precisely positioned within $\pm 0.1 \mathrm{~mm}$ and maintain positional stability under thermal fluctuations. Thermal expansion and contraction of lead tiles would shift the tile pattern and directly translate into gamma ray burst position errors. The flatness of the tile plane had to be within $\pm 0.25 \mathrm{~mm}$ and the entire pattern was to be parallel with the detector array pattern within 0.5 arc-minutes.

The engineering requirements were stringent as well. The mask assembly was allocated just $21.5 \mathrm{~kg}$ and required to have a minimum natural frequency greater than $50 \mathrm{Hert} z$ with a very low thermal expansion. The elimination of any trace of excess adhesive in untiled areas proved to be one of the most difficult requirements to meet. Generally, excess adhesive around the perimeter of mating surfaces, or "squeeze-out," is a sign of a good bond. Restricting the adhesive flow beyond the footprints of any of the $\sim 50,000$ lead tiles was a huge challenge.

Multi-layer insulation blankets on the mask that would have helped minimize the temperature extremes had to be reduced to prevent attenuating X-rays. As a result, the mask had to be designed to withstand temperatures from $-86^{\circ} \mathrm{C}$ to $+60^{\circ} \mathrm{C}$. This magnified differential thermal expansion issues between the lead tiles and the mask panel. Since lead has a relatively high coefficient of thermal expansion and the panel was designed for near-zero expansion, swings in temperatures could have caused severe bowing of the mask if the lead tiles were rigidly adhered to the panel.

\section{BAT's Coded Aperture Mask Concept Development}

\subsection{Initial mask fabrication concepts}

An extensive development effort was necessary to verify the techniques and procedures used to meet the strict requirements specified for the mask. Consequently, the design of the coded aperture mask and the research and development of the manufacturing process were conducted in parallel. Throughout the development process, various manufacturing concepts were evaluated and multiple trade studies were performed before determining the best overall fabrication approach.

Due to the $1.2 \times 2.4 \mathrm{~m}$ size of the mask, bonding $\sim 50,000$ lead tiles to the surface accurately was no small task. Several options were not seriously considered because they could not meet science or design requirements. Certainly, hand placement of tiles was a last resort. The most obvious concept was to bond a large sheet of lead to the panel and machine the pattern directly out of the lead. Machining lead created an abundance of lead particulates, which were impossible to remove completely from the substrate. Metallic dust was a contamination concern and violated the science requirement prohibiting metallic material in untiled areas. The desired corner radius of each untiled location demanded a small tool, making machining extremely tedious by requiring multiple tools and/or tool passes. Lastly, the thermal expansion effects of this monolithic lead sheet would bow the honeycomb panel and cause unacceptable pointing errors. 
Clearly, alternatives had to be reconsidered and one option stood out from the rest. The concept was to place all the tiles in a "tile tool" to control the pattern while adhesive was applied to the substrate and lowered to contact the tile surface. After the adhesive cured, the panel was to be lifted up and, ideally, all the tiles would be bonded to the substrate. Getting this concept to work required many tests and trades.

One of the first trade studies involved selecting the adhesive to bond the lead tiles to the honeycomb panel. Because lead has a high coefficient of thermal expansion $\left(29 \times 10^{-6} / \mathrm{K}\right)$ and the panel had a near zero CTE, a very stiff bond would cause severe bowing of the mask, if not outright failure. A soft or flexible adhesive allowed the tiles to float as they expanded and contracted. After an extensive survey of candidates, Nusil's CV10-2568 silicone rubber adhesive was selected. The required thickness of the adhesive was calculated to be about $0.8 \mathrm{~mm}$ in order to provide enough flexibility to the interface.

Another trade study focused on the best tile tool design. Three options were developed that merited consideration. Miniature samples were made to verify the feasibility of each alternative. The following sections describe the advantages or disadvantages associated with each option.

\subsection{Option 1: punched tile template with featured tiles}

Option 1: Punched Tile Template

This option consisted of using lead tiles with small protrusions on one side that would fit into cutouts on a large tile tool. It became obvious that maintaining flatness of the tile array using this type of tile fixturing would be very difficult. Custom tooling was generated to produce the desired clover cut in a thin sheet of metal. The punching process severely distorted the flatness of the template, especially at the cut. Efforts to improve the flatness by varying sheet thickness, material, and machine parameters were less than effective. Although an iterative process may have yielded an improved punch and die, it was difficult to estimate the additional cost and time required to achieve an acceptable template. Machine travel

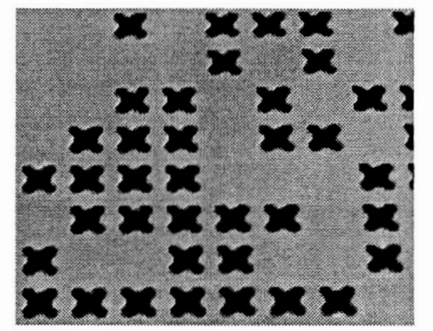

limitations required that the sheet be punched, repositioned, and punched on the other side to fabricate a single template with the entire tile pattern. Repositioning complicated the programming and introduced a tile-to-tile misalignment risk if the sheet was not perfectly replaced.

\subsection{Option 2: machined 4-hole tool with featured tiles}

Option 2 was the same basic design principle as option 1 without the flatness, travel, or programming complications. The basic tool design incorporated 4 machined holes for each of the $>50,000$ tile positions in the specified random pattern. The tiles were designed with two protrusions to provide position and clocking. The additional two holes in the tool allowed tiles to be positioned in any of the 4 in-plane rotations, which simplified tile installation. Another advantage over option 1 was that no special tooling was required for fabrication.

\subsection{Option 3: machined pocket tool with flat tiles}

The basic concept behind option 3 was to place flat tiles in a tool with machined pockets. Flat tiles were placed in individual pockets, which would provide in plane positioning and flatness. In large pocketed areas, machining one region would have eliminated the ability of the tool to accurately locate the tiles. The tolerance stackup of several small tiles in a row would have produced an inconsistent tile pattern. Consequently, it was important to leave enough material in each corner of the pockets to restrict the tile from shifting or rotating. Tiles could be easily installed in the tooling by sliding them into position since there were no features at risk.

Option 2: Machined 4-Hole Tool

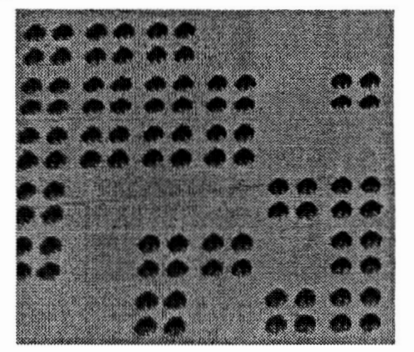

Option 3: Machined Pocket Tool

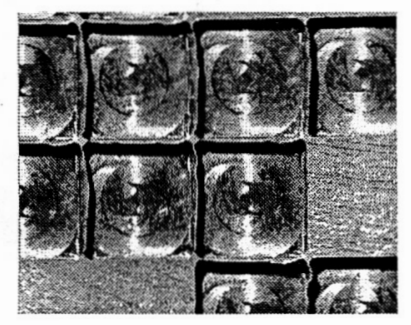




\subsection{Option 2 \& 3 comparison}

Based on favorable results in the $5 \times 5 \mathrm{~cm}$ development samples, options 2 and 3 were selected for further consideration. In order to better evaluate the pros and cons of each option, $15 \times 15 \mathrm{~cm}$ tooling, flat \& featured tiles, and new test samples were generated. Using these samples as a comparison, a detailed trade study was performed. The purpose of this trade was to determine which option would better meet the science and design requirements within the cost and schedule constraints.

Throughout the tile and tool manufacturing process, cost and time were tracked and recorded. In addition, design parameters, possible improvements, and potential scaling concerns were noted for future reference. Each concept was carried out from tile and tool design to the bonding of tiles to the substrate. Since both options produced adequate samples, the two main differences to consider were tile and tool design from a fabrication standpoint.

\subsection{Tool design - pocket vs. 4-hole}

Based on a fabrication comparison of the $15 \times 15 \mathrm{~cm}$ sample tooling, option 2 had many advantages. The machined pocket tool was twice as expensive and took twice as long to fabricate as the 4-Hole tool. Obviously, drilling a pattern of 4 tiny, shallow holes in each tiled location required much less machining time than generating an acceptable pocket.

Science and design requirements drove tight tolerances on the position and size of each pocket. A small corner radius was necessary to assure easy tile insertion. Since proper tile array flatness was a function of the pocket depth and floor flatness, these features were critical. A plunge cut was used to remove the bulk of the material and the outer edge of the pocket floor was machined to provide the desired tile and array flatnesses.

At the minimum tile size and the maximum pocket size, the gap between tiles could be no more than $0.1 \mathrm{~mm}$. Machining the tooling to meet these requirements left a thin, foil-like wall between adjacent pockets. These walls were easily deformed so they had to be removed by hand. Any particulates in the tool could offset the tile position and overall flatness of the tile plane.

The requirements of the 4-Hole tool were much less involved, which led to simplified programming and fabrication. Four identical holes were machined at equal distances from the center of each tile location specified in the pattern. This tool design eliminated the need for multiple passes, complex tool paths, and tool changes, other than for tool wear or damage. Since there were no thin walls

Featured Tiles Bonded to Substrate

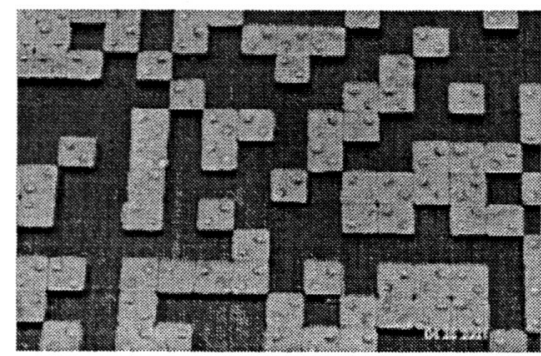

Flat Tiles Bonded to Substrate

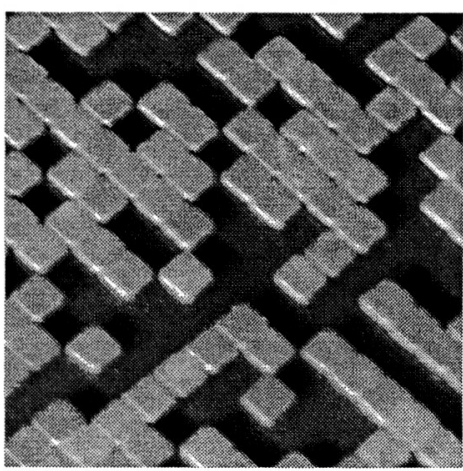
produced, only minimal by hand operations were needed for cleanup. From a tool design standpoint, the 4-Hole tool concept was by far the better alternative.

\subsection{Tile design - flat vs. featured}

Conversely, option 3 was by far the better alternative when considering tile fabrication. The production costs for featured tiles were at least twice as high as flat tiles. Flat tiles could be produced quickly and easily through a simple punching operation. Standard punch and die tooling were used as opposed to the custom featured tooling required for featured tile production. The lack of features also greatly reduced the handling, packaging, and shipping risks, which lowered the overall tile production costs significantly. Flat tiles were more durable which allowed for increased flexibility in the methods considered for bonding tiles to the substrate.
Flat Lead Tiles in Tool Pocket

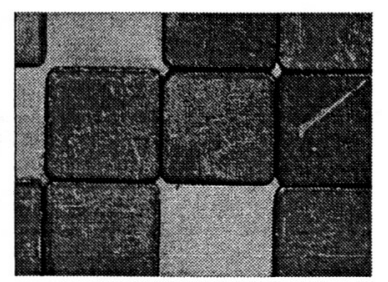


The featured tiles were fabricated by hand, one at a time, with a custom punch and featured die. Generating these featured tiles was very time consuming and it produced tiles of extremely low quality. Uniform tile flatness was a definite concern. A slight reduction in tile weight could be achieved by using tiles with half moons, however, this may have further complicated the design of the custom tooling.

Although automating this process would have minimized production time, the handling, packaging, and shipping risks to the tile features were even more likely. Since the tiles were costly to generate, an abundance of replacement tiles would have driven the cost even higher.

Another consideration was tile weight. Flat tiles weighed slightly less than featured tiles. The small lead protrusions accounted for a minor difference in the individual tile weight, but multiplied by 50,000 tiles resulted in a significant difference in weight. The features also made tile placement far more difficult. Flat tiles were easily slid into a pocket, without risk of damage, twice as fast as populating featured tiles into a tile tool. Since populating an entire tile tool took hundreds of hours, the cost savings were substantial.

\subsection{Concept comparison results}

Both concepts produced acceptable $15 \times 15 \mathrm{~cm}$ samples. Based on the results, either manufacturing option was likely to produce an acceptable Flight Mask, however, a choice had to be made. The flat tiles were selected for a number of reasons. Flat tile production could begin immediately. Not only did this remove the schedule impact required to develop featured tile custom tooling, it also facilitated bonding development because flat tiles were readily available. Flat tiles also reduced the number of labor hours required to populate a tile tool considerably. Future projects with the luxury of more development time may find featured tiles to be more desirable.

\section{Lead Tile Bonding Process Development}

The concept evaluation and lead tile bonding process were conducted in parallel. As discussed, the flat tiles were easily produced which facilitated test sample bonding and process improvement. Each successive test sample was an opportunity to explore panel to tile bonding techniques, improve tile adhesion, and ultimately minimize the squeeze-out of excess adhesive into untiled areas.

In the design phase, CV10-2568 silicone adhesive had been selected to minimize the large coefficient of thermal expansion, CTE, gradient between lead and the composite substrate. The idea was that the lead tiles would float on a thick bondline, a minimum of $0.5 \mathrm{~mm}$, of silicone adhesive. The greatest challenge in the bonding process was attaining a sufficient bond between pre-cured tiles and the panel without allowing any squeeze-out beyond the tile footprint.

Ideally, bonding the tiles to the substrate in a single operation would avoid variations in flatness, tile alignment, and squeeze-out between multiple bonded regions. Simply applying wet silicone to the substrate and lowering the panel to the tiles produced less than favorable results. A $0.8 \mathrm{~mm}$ thick template was used to coat the substrate with adhesive in the tiled areas but the silicone flowed under the template. As mixed, it was virtually impossible to control the flow of wet silicone precisely. After using Cab-o-sil as a thickening agent, the adhesive still squeezed into untiled regions when adequate bonding pressure was applied.

After many bonding iterations and process improvements, the technique developed for bonding the tiles to the substrate incorporated two separate bonding efforts. A thick layer of CV10-2568 silicone was "pre-cured" on one surface of each lead tile. Later, these pre-cured tiles were bonded to the panel by using a wet layer of small CV10-2568 silicone dots applied to the substrate, which will be discussed in detail in another section. 


\subsection{Lead tile pre-curing process}

1. Over 100,000 Flight quality lead tiles were fabricated at Johns Hopkins University Applied Physics Lab (JHUAPL).

2. Lead tiles were prepared by ultrasonic acetone and alcohol cleaning in batches of 1000. Extensive trade studies and trials were performed which led to this technique.

3. A "skim coat" of adhesive was applied to tiles using a sequence of steel templates. Steel templates were located tightly with respect to each other by a magnetic chuck.

4. The first template located 901 tiles in a grid pattern. Tiles were slid into square openings in the template.

5. Once in the grid, tiles were primed by spraying CF2-136 silicone primer and allowed to air dry.

6. The second template was pinned over the grid of tiles to center a $4 \times 4 \mathrm{~mm}$ square opening on each tile. This opening provided the location of the pre-cured adhesive.

7. Up to four grids were placed on a magnetic chuck, which provided a tight seal between the lead tile surface and the template. This was an essential step in eliminating excess adhesive beyond the perimeter of the tile.

8. CV10-2568 silicone adhesive with $3 \%$ Cab-o-sil was smeared over the template to fill in the openings. This template controlled the pre-cured adhesive thickness on the tiles.

9. After curing at room temperature for 2-4 hours, a second skim coat of CV10-2568 without Cab-o-sil was applied to generate a smooth surface finish.

10. The adhesive was cured to the tiles in an oven at $150^{\circ} \mathrm{F}$ for a minimum of 4 hours.

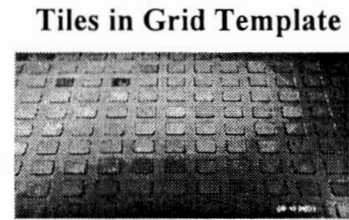

Primed Tiles in Grid

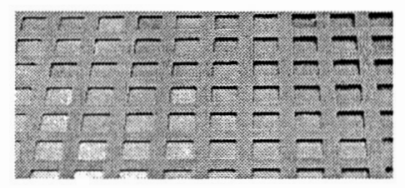

Pre-cured Flat Lead Tile

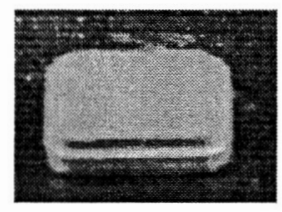

\subsection{Tile tool populating}

After a sufficient number of pre-cured tiles were fabricated, the next step was to install each tile into a pocket on the tile tool. This was done by hand and took several hundred hours. Unfortunately, a significant percentage of the pockets had to be de-burred or opened with a special gage block to allow the tile to seat properly. Any debris left in the pockets of the tool could result in unseated tiles.

Periodically, the seated tiles were inspected to make sure that none had become dislodged or tipped in the pocket. A single tile wedged at an angle between the tool and the substrate could be catastrophic. A tile out of its pocket or even just raised on one edge could result in thousands of unbonded tiles in the surrounding areas.

Populating the Tile Tool from the Center Outward

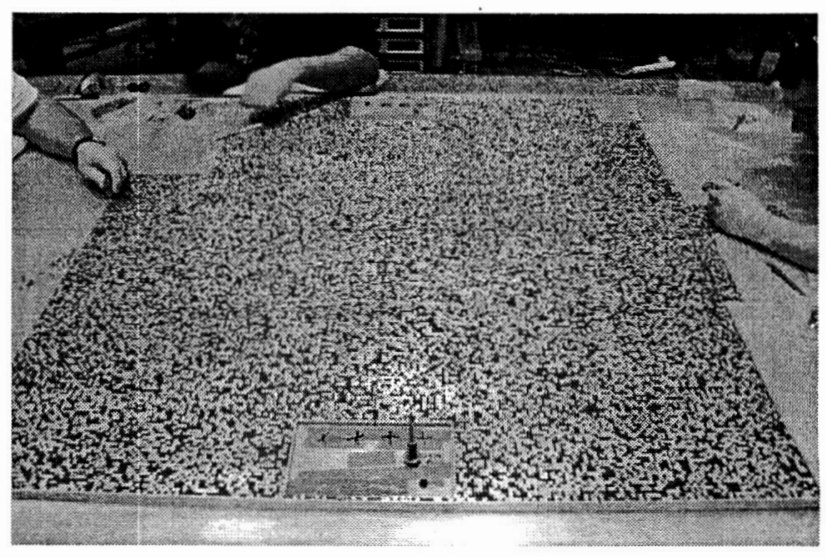

\subsection{Wetting the panel for bonding}

After many tests, a technique for using a "skim template" to precisely locate adhesive dots on the panel surface was perfected. Template material was a key since it had to conform closely to the substrate surface to avoid forcing adhesive into gaps. A single $0.2 \mathrm{~mm}$ thick G10 template was machined with $4 \mathrm{~mm}$ diameter holes in the desired pattern. After the pre-cured tiles were installed in the tile tool, the template was positioned on the substrate with pins.

Wet CV10-2568 with 3\% Cab-o-sil was applied to the center of skim template. The adhesive was scraped from the center out toward the edges until a uniform coat was achieved. The template was removed by carefully lifting one edge off of the panel and slowly working toward the other end leaving a pattern of wet adhesive dots on the surface, without smearing adhesive into the untiled areas.

Skim Template on Panel

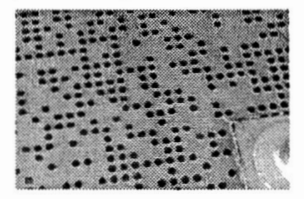

Wet Adhesive on Panel

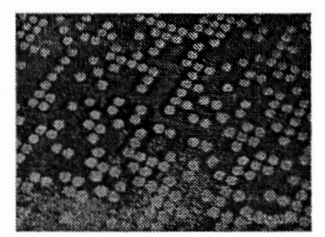




\subsection{Mating the panel and pre-cured tiles}

The next crucial step in the bonding process was aligning the wet adhesive dots on the panel with the pre-cured surfaces of the tiles in the tool and mating them in a steady and precise way to avoid smudging the adhesive or dislodging tiles. This was far too delicate of an operation to be performed by hand. As discussed, even one tile accidentally shifted out its pocket could lead to catastrophic results.
Flight Panel with Lowered with Actuator System

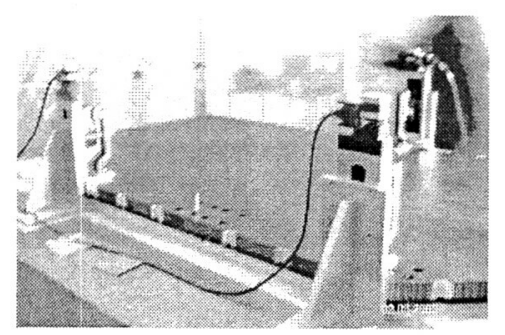

Kinematic Interface

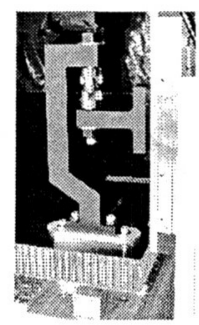

A three-point linear motion assembly was designed to lower the panel slowly and parallel to the tile tool within 0.005 $\mathrm{mm}$. The device consisted of three motorized encoded linear motion actuators that were synchronized and controlled by computer. Aluminum brackets were mounted to the top surface of the panel, which kinematically interfaced with the actuators using a ball-cone-groove-plate arrangement. Two pins were installed through alignment holes the panel and tile tool to maintain position once the panel was completely supported by the tiles. The actuator system worked extremely well. The weight of the lead exceeded the actuator system capability. Consequently, the interfaces at the brackets were used to raise the panel by hand from the tile tool after bonding.

\section{Qualification Panel Bonding Development}

Although the $15 \times 15 \mathrm{~cm}$ samples were very effective for identifying parameter changes, implementing improvements, and validating the results, scaling up to the Flight bond was a major concern. In an effort to avoid unexpected difficulties during the full Flight Mask bond, a half size Qualification panel, was developed. This Qualification panel was used to verify that techniques and procedures developed on the smaller samples would work on a much larger scale. The main areas of interest were tile positional accuracy, bond area, bond quality, and excess adhesive in untiled areas.

There were actually two bonds performed as part of the bonding qualification procedure. The second was necessary after an unsuccessful first attempt. The adhesive was removed from the panel and the same bonding surface was prepared for the second effort. There were many lessons learned during the evaluation of the initial qualification bonding procedure. Three main process modifications resulted in an overwhelmingly successful second attempt.

\subsection{Qualification panel design and tooling}

The Qualification panel was fabricated to the exact requirements of the Flight panel designed by Swales. ${ }^{3}$ Both panels were fabricated at Composite Optics International (COI) in San Diego, CA. The panels consisted of Korex core $38.1 \mathrm{~mm}$ thick sandwiched between two $0.5 \mathrm{~mm}$ thick graphite-cyanate ester facesheets. The facesheets were comprised of a quasi-isotropic layup, [0,45,90,-45]S of M60J fibers prei-mpregnated in a 954-3 resin matrix. The University of Maryland perforated the core. In an effort to minimize costs and schedule impact, the Flight tooling was divided into 3 pieces, the center and two "wings." The middle region, a $1.22 \mathrm{~m}$ square, was used for the Qualification bond tooling. To facilitate the schedule, this portion of the tool was fabricated first and the wings were fabricated during the Qualification bonds.

\subsection{First qualification bond}

Multiple flatness inspections of the panel's bonding surface yielded a maximum peak to valley of $0.3 \mathrm{~mm}$. To ensure contact between the tiles and the wet adhesive on the panel, flatness mappings were used to tailor the pre-cured adhesive thickness to accommodate panel peaks and valleys. Templates controlling pre-cured tile thickness were limited by steel thickness availability. Consequently, three pre-cured tile thicknesses were used in the tile tool to compensate for the panel contours. The low, mid-level, and high areas of the panel were populated with $0.76,0.58$, and $0.46 \mathrm{~mm}$ thick pre-cured adhesive on the tiles, respectively.

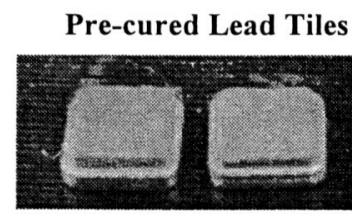

$0.76 \& 0.46 \mathrm{~mm}$ Pre-cured CV10-2568 
Unfortunately, the tile thicknesses may have overcompensated for the contour of the panel. Placing two different thickness tiles in close proximity on the panel created a "stepped effect" instead of a smooth transition. There were jumps of $0.18 \mathrm{~mm}$ or $0.13 \mathrm{~mm}$ wherever tiles of two different heights were adjacent to each other.

Since the thickness of the wet adhesive was only $0.2 \mathrm{~mm}$ and the maximum difference in height between pre-cured tile thicknesses was $0.18 \mathrm{~mm}$, there was only a margin of $0.02 \mathrm{~mm}$. Consequently, the tile tool and panel contours had to match exactly for the coarsely stepped ( 3 steps over $0.3 \mathrm{~mm}$ ) tile tool to work properly, given the panel stiffness and low bonding pressure.

The $15 \times 15 \mathrm{~cm}$ samples were bonded successfully with almost no pressure but this was one of the issues that arose in scaling up to the larger panel. Evenly distributing $215 \mathrm{~kg}$ over the $1.5 \mathrm{~m}^{2}$ surface resulted in approximately $1380 \mathrm{~Pa}(0.2$ psi) of bonding pressure. Increasing the bonding pressure increases the panel conformance to the tool, the pre-cured adhesive compression, and the wet adhesive compression. With the low bonding pressure, the wet adhesive only compressed a few hundredths of a millimeter on the thick tiles that bonded, resulting in an extremely low panel conformance to the tile tool.

Based on the principle that the pre-cured tiles were compensating for panel flatness, the $0.2 \mathrm{~mm}$ thick skim coat on the panel surface was designed to accommodate the tile-to-tile pre-cured adhesive thickness variations only. The skim coat was too thin to accommodate any mismatch in the contours at the low pressure

\subsection{Bonding theory}

As a result of the first qualification test, a simple formula was defined. It was developed to theoretically determine if a successful bond could be achieved, based on the given parameters. The formula can be expressed as

$\begin{array}{lll}\mathrm{H}+\mathrm{C}>\mathrm{F}_{\mathrm{T}}+\mathrm{F}_{\mathrm{P}}, \text { where } & \begin{array}{l}\mathrm{H}=\text { Height of wet dot, } \\ \mathrm{C}=\text { Conformance panel to tile tool, },\end{array} & \begin{array}{l}\mathrm{F}_{\mathrm{T}}=\text { Flatness of populated tile tool (Peak to valley), } \\ \mathrm{F}_{\mathrm{P}}=\text { Flatness of panel (Peak to valley) }\end{array}\end{array}$

Assuming a "peak" on the tile tool touches a "peak" on the panel, the maximum possible gap between surfaces occurs. For a successful bond, the combination of the wet adhesive height and the panel conformance must overcome this gap. The conformance variable (C) is a combination of panel deflection and pre-cured adhesive compression. Conformance is a function of bonding pressure, panel stiffness, and the compressive modulus of the pre-cured adhesive. Obviously, the easiest variable to control is the wet adhesive height $(\mathrm{H})$. For this application, the boundary conditions were that the wet adhesive height could not be compressed to zero and excess adhesive squeeze-out beyond the tile perimeter was not permitted.

\subsection{Second qualification bond}

In summary, three main process changes led to a successful qualification bond on the second attempt. The pre-cured thickness variations were eliminated and the tool was populated uniformly with $0.58 \mathrm{~mm}$ thick precured tiles. The height of the wet adhesive dot was increased to $0.48 \mathrm{~mm}$. The diameter was reduced to $1.3 \mathrm{~mm}$ to maintain a constant volume in an effort to avoid squeeze-out in untiled areas. The bonding pressure was increased to about $14,000 \mathrm{~Pa}$ ( $2 \mathrm{psi}$ ) using a vacuum bagging technique, which provided an adjustable uniform pressure.

The pressure and the wet adhesive geometry were the variables adjusted to Second Qualification Bond

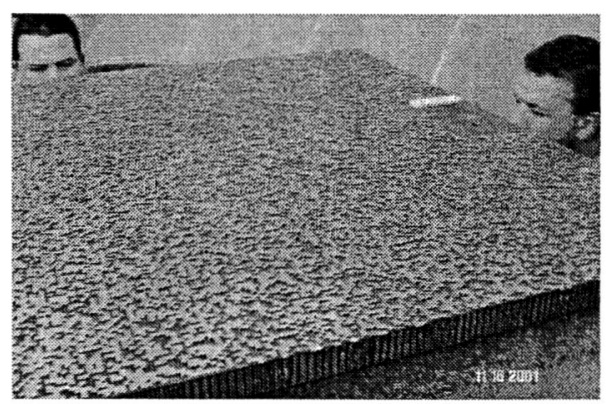
ensure tile adherence without excessive squeeze-out in untiled regions. A smudge test was developed to gauge the amount of tile contact with the panel at a given pressure. The smudges were generated on the bonding surface by compressing the panel against the pre-cured tile adhesive. Using smudge test pressures and skim coat criteria, test samples were fabricated to check squeeze-out results prior to bonding full size panels. 


\section{Testing of Coded Aperture Mask}

After proving the bonding concept on the qualification panel, several tests were performed to prove that the tiles would remain in place in the rocket launch and space environments. An acoustic test was performed on the panel simulating the rocket launch vibration environment. The panel was suspended in NASA Goddard's large acoustic chamber and blasted with up to 130 decibels of acoustic energy, creating about 20 G's of acceleration on the lead tiles. The mask passed without any loss of tiles. Also a populated $30 \times 56 \mathrm{~cm}$ coupon was random vibration tested to the limit of the vibration table, about $21 \mathrm{G}$ 's. The coupon passed the test.

A long term thermal cycle test was run to prove that the CV10-2568 adhesive was the correct choice to accommodate differential thermal expansion due to temperature swings on orbit. The concern was that the adhesive might fatigue after many cycles, or that it would fail to work at low temperatures. A $15 \times 15 \mathrm{~cm}$ panel with about 500 lead tiles was fabricated and thermal cycled. The coupon was cycled over 3000 times in several different temperature ranges, between $-55^{\circ} \mathrm{C}$ and $20^{\circ} \mathrm{C}$. Due to an error in the test setup, the coupon was exposed to $-150^{\circ} \mathrm{C}$. The tiles remained in place in all cases, thus validating the choice of adhesive.

These tests gave the project confidence to proceed with populating the BAT's flight mask. Once the lead tiles were bonded to the flight mask, the mask passed an acoustic test at a lower workmanship level. Finally, the mask was ready for assembly. After alignment and integration, the Flight Mask, as part of the BAT structure, completed several sine burst and random vibration tests, all without incident. Preparations are currently underway for a thermal vacuum test on the BAT structure.

\section{Conclusions}

After two years of intense research, trade studies, and process improvement, procedures were developed to meet the challenges of building a large coded aperture mask. Using two bonding procedures and various techniques described here, a $2.4 \times 1.9 \mathrm{~m}$ substrate was successfully bonded with more than 50,000 tiny lead tiles, without squeeze-out in any untiled areas.

The Flight panel has undergone extensive testing to prove that the stringent $\mathrm{BAT}$ requirements have been met. The testing of the BAT structure is well underway. The BAT team is confident in the future performance of the largest coded aperture mask ever built for the Burst Alert Telescope in fulfillment of the Swift Mission.
Flight Mask Acoustic Test

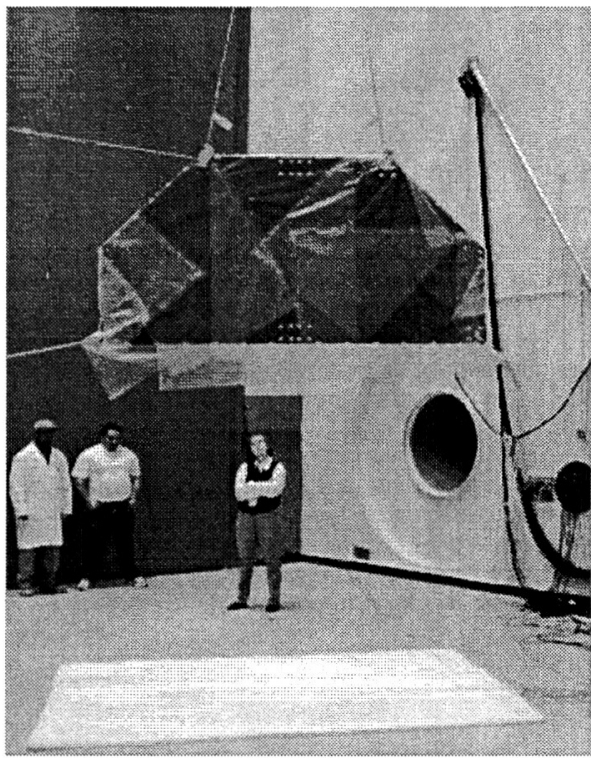

\section{$2.6 \mathrm{~m}^{2}$ Flight Mask Bonded with $>50,000$ Tiles $^{5}$}



Flight Mask Assembled on Mask Support Structure






\section{ACKNOWLEDGEMENTS}

NASA/GSFC Lead Technician, Mike Schoolman; Code 543, Code 547, Code 541, Code 549, and the BAT Team; Swales; Mantech; COI; JHUAPL; U of MD; and QSS.

\section{REFERENCES}

1. The Swift website can be found at http://swift.gsfc.nasa.gov/

2. An excellent source on coded aperture mask literature is: http://heawww.gsfc.nasa.gov/docs/cai/coded_intr.html 3. INTEGRAL's masks are detailed at: http://pollux.uv.es/integral/Masks/integralhome.htm

4. Rodini, Ben, "Development of a Lead-Coded Composite MASK for Gamma-Ray Detection," 34 ${ }^{\text {th }}$ Int'l SAMPE Technical Conference, to be held November, 2002.

5. NASA News Release: 02-044, 3/13/02, NASA Gamma-Ray Burst Satellite Team Assembles World's Largest Telescope Mask.

*danielle.vigneau@gsfc.nasa.gov; phone (301) 286-6099; fax (301) 286-1658; http://www.gsfc.nasa.gov/; NASA/GSFC, Greenbelt, MD 20771;

**drobinson@mscmail.gsfc.nasa.gov; phone (301) 286-9926; fax (301) 286-1658; http://www.gsfc.nasa.gov/ 